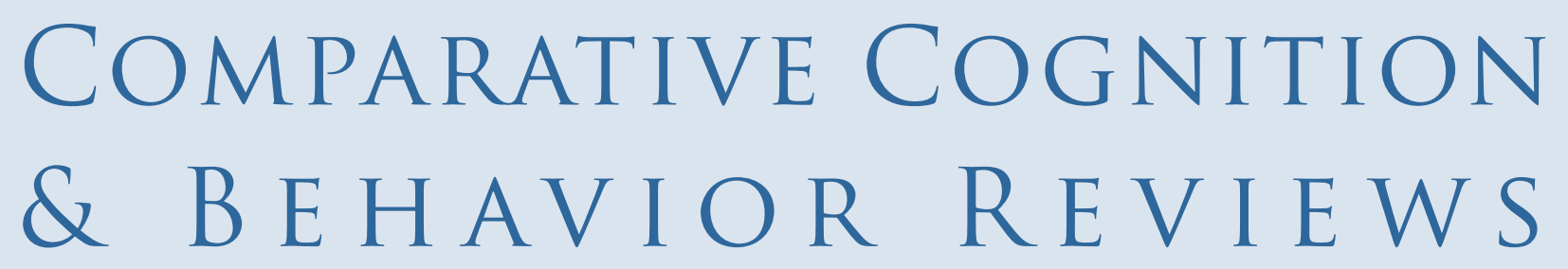

\title{
Is the Susceptibility to Visual Illusions Related to the Relative Brain Size? Insights from Small-Brained Species
}

\author{
Alessandra Pecunioso \\ and Maria Santacà \\ Department of General Psychology \\ University of Padova
}

\author{
Maria Elena Miletto Petrazzini \\ Queen Mary University of London
}

\author{
Christian Agrillo \\ Department of General Psychology \\ Padua Neuroscience Center \\ University of Padova
}

Visual illusions are powerful tools to understand similarities and differences in the perceptual mechanisms of human and nonhuman animals. Such investigation is particularly important in the presence of animal species whose brains largely differ from ours, because it can reveal whether perceptual laws described in humans are strictly related to the peculiarity of large brains, as the case of mammals and birds. Here we review the literature on visual illusions in species with a much smaller relative brain size. Most works on this subject have investigated fish, whereas only a few studies have been conducted on amphibians and reptiles. Taken together, the existing literature found more similarities than differences in the perceptual mechanisms underlying size, numerosity, brightness, motion, and subjective contours among vertebrates, regardless of the high variability in the relative brain size of the species.

Keywords: fish, reptiles, amphibians, visual illusions, Gestalt, comparative perception

\section{Introduction}

The study of visual illusions represents a useful tool in different research fields. Visual illusions are commonly used to establish the perceptual mechanisms underlying our perception of static and dynamic events (for a review, see Eagleman, 2001; Gregory, 1997). Visual illusions have become a valid tool in clinical psychology as a noninvasive screening for detecting schizophrenic and other psychopathological traits (e.g., Gori, Molteni, \& Facoetti, 2016; Notredame, Pins, Deneve, \& Jardri, 2014; Pessoa, Monge-Fuentes, Simon, Suganuma, \& Tavares, 2008). Last, they represent a powerful set of stimuli to compare the vision of human and nonhuman animals. With respect to this issue, the past two decades have been characterized by a growing number of studies finding that apes, old-world monkeys, and new-world monkeys are susceptible to many visual illusions. Nonhuman primates perceive size illusions (e.g., chimpanzees, Pan troglodytes: Parrish \& Beran, 2014; capuchin monkeys, Sapajus apella: Parrish, Brosnan, \& Beran, 2015; Suganuma, Pessoa, Monge-Fuentes, Castro, \& Tavares, 2007), depth illusions (baboons, Papio papio: Barbet \& Fagot, 2002), orientation illusions (rhesus monkeys, Macaca mulatta: Agrillo, Parrish, \& Beran, 2014b), numerosity illusions (rhesus monkeys: Beran, 2006), and motion illusions (rhesus monkeys: Agrillo, Gori, \& Beran, 2015). 
The evidence that nonhuman primates exhibit humanlike perception of many illusory phenomena clearly showed that the perceptual laws commonly studied in psychological textbooks are not only a human prerogative. However, these results may not be surprising per se, given the similarities in the brain between human and nonhuman primates. The fact that birds are also susceptible to different illusory phenomena has made the picture more complex. For instance, today we know that domestic chicks (Gallus gallus) perceive the Ebbinghaus illusion (Rosa Salva, Rugani, Cavazzana, Regolin, \& Vallortigara, 2013), pigeons (Columba livia) perceive the Ponzo illusion (Fujita, Blough, \& Blough, 1991), and parrots (Psittacus erithacus) perceive the Müller-Lyer illusion (Pepperberg, Vicinay, \& Cavanagh, 2008). The evidence that birds and humans seem to share similar perceptual biases in size estimation is intriguing because birds and primates are certainly characterized by different brain structures. However, among birds, cases of reverse illusions have been also reported (i.e., the perception of a visual illusion in the opposite direction than that perceived by human observers). For instance, pigeons and bantams (Gallus gallus) perceive a reverse Zollner illusion (Watanabe, Nakamura, \& Fujita, 2011, 2013); pigeons also experience a reverse Ebbinghaus illusion (Nakamura, Watanabe, \& Fujita, 2008). Hence, the comparison between humans and birds provided mixed results in terms of similarities in the perceptual mechanisms underlying vision, leaving open the possibility that the majority of perceptual laws described in our species are mainly confined to mammals.

Mammals and birds differ from each other with respect to relative brain size; the formers has a higher brain size compared with body size (Jerison, 1991, 1994; van Dongen 1998). One may hypothesize that the presence of the complex perceptual mechanisms underlying illusory phenomena is somehow related to the complexity of the brain. Indeed, increases in brain size have often resulted in increases in the number of neural centers, have resulted in a higher number of neuronal cell classes within each neural center, and are supposed to be positively correlated to qualitative differences in behavioral complexity (Northcutt, 2002).

Author Note: Alessandra Pecunioso, Department of General Psychology, Via Venezia 8, 35131 Padova, Italy.

Correspondence concerning this article should be addressed to AlessandraPecunioso at alessandra.pecunioso@studenti.unipd.it
To shed light on the correlation between relative brain size and susceptibility to visual illusions, we need to enlarge the range of species investigated, encompassing more distant-related species to humans. Although mammals and birds are often called "higher" vertebrates, fish, amphibians, and reptiles are commonly defined as "lower" vertebrates (Boehm, Iwanami, \& Hess, 2012; Chianese et al., 2011; Sneddon, 2004) and are characterized by a smaller relative brain size. Fish and amphibians, in particular, are the vertebrate groups with the smaller index (Jerison, 1994). With respect to reptiles, even though birds are reptiles too, birds have brains that are 6 to 10 times larger on average than those of nonavian reptiles with the same body size (Northcutt, 2002). If lower vertebrates show a similar susceptibility to visual illusions of mammals and birds, then the hypothesis that the perceptual mechanisms underlying visual illusions primarily emerge in brains with a high degree of size/complexity could not be supported.

In this work, we review the existing literature on the field by dividing the literature of lower vertebrates into fish, amphibians, and nonavian reptiles. As many cartilaginous fishes have brains as large as those of mammals and birds (Bauchot, Platel, \& Ridet, 1976; Demski \& Northcutt, 1996; Northcutt, 1997), this review is limited to teleost fish, thus excluding the research on visual illusions in bamboo sharks (Chiloscyllium griseum: Fuss, Bleckmann, \& Schluessel, 2014; Fuss \& Schluessel, 2017).

\section{VISUAL ILLUSIONS IN TELEOST FISH} Freshwater Fish

To date, most studies have investigated the illusory perception of freshwater fish, mainly because maintaining freshwater aquaria is often easier and cheaper than maintaining saltwater aquaria. Therefore, freshwater aquaria are often more frequent in comparative psychology laboratories.

\section{Size Illusions}

One of the most popular geometrical illusions is the Müller-Lyer illusion (Figure 1a). In its classical configuration, two lines are presented, one of which ends with two pairs of arrowheads pointing inward (Figure 1a, upper line) while the other ends with two arrowheads pointing outward (Figure 1a, lower line). Humans typically believe that the line with the arrowheads facing inward is longer than the other. According to Gregory (1963), we perceive the line with the

COMPARATIVE COGNITION \& BEHAVIOR REVIEWS 
(a)

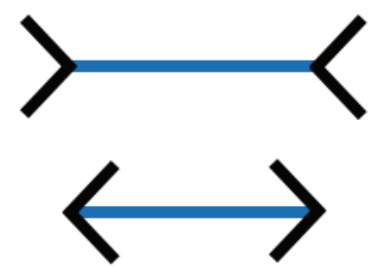

(b)

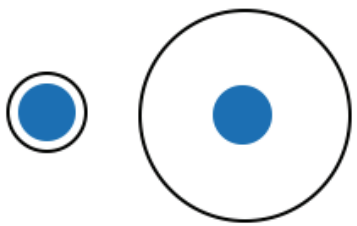

(c)

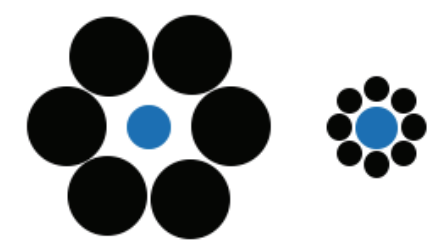

Figure 1. Size illusions investigated in lower vertebrates. (a) Müller-Lyer illusion: Although the two blue lines are identical, the line with the arrowheads

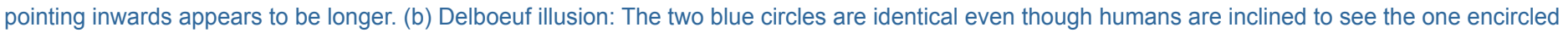
by the smaller ring as larger. (c) Ebbinghaus illusion: The two blue circles are identical, but the one surrounded by smaller inducers appears larger to human observers.

inward-pointing arrows as being farther in depth, and we perceive the line with the outward-pointing arrows as closer in depth (Ward, Porac, Coren, \& Girgus, 1977). Because of the size-constancy mechanisms often involved to compensate for the decreasing retinal size of an item with increasing distance, the line with the inward-pointing arrows appears longer. Alternatively, because we are inclined to believe that the inward-pointing arrows indicate longer lines, Howe and Purves (2005) suggested that the Müller-Lyer illusion is the result of a probabilistic strategy of visual processing.

Sovrano, Da Pos, and Albertazzi (2016) investigated this illusion in redtail splitfin (Xenotoca eiseni). The authors used social reward to train the subjects: The focal fish was removed from its social group and placed in an unfamiliar environment. To rejoin its social companions, it was required to pass through one of two identical tunnels placed at opposite corners. Each tunnel was associated with a line. Only the door associated with the reinforced stimulus (the longer line for $50 \%$ of the subjects) allowed the focal fish to rejoin the conspecifics. In the test phase, the authors presented the Müller-Lyer illusion. Redtail splitfin showed susceptibility to the illusion, as they selected the tunnel associated with the line appearing longer or shorter (depending on which stimulus was reinforced).

Evidence of a Müller-Lyer illusion in fish was recently found in another teleost freshwater fish, the guppy (Poecilia reticulata: Santacà \& Agrillo, 2020). The subjects were initially presented with two lines differing in length and were required to reach the longer one in order to obtain a piece of commercial food flake. In the test phase with the illusory configuration, guppies selected the line associated with inward arrowheads, showing a humanlike perception.
Another important geometrical illusion is called the Delboeuf illusion. This illusion occurs when we misperceive the size of two otherwise identical objects when they are encircled by a smaller and a larger ring (Figure 1b). Both assimilation and contrast effects seem to play an important role: When the ring is far from the object, the ring is perceived to contrast, leading to an underestimation of the object size. On the contrary, when the ring is close to the object, the object is supposed to assimilate to the ring, and the object itself appears to be larger (King, 1988). Recently, Lucon-Xiccato, Santacà, Miletto Petrazzini, Agrillo, and Dadda (2019) found evidence that guppies perceive the Delboeuf illusion by using two methodological approaches. In one experiment, the spontaneous behavior in the presence of different amounts of food was observed. There is indeed evidence that guppies try to maximize food intake and reach for the larger amount of food when given the possibility to select a large and a small piece of food (Lucon-Xiccato, Miletto Petrazzini, Agrillo, \& Bisazza, 2015). Two pieces of food were circumscribed by rings drawn on the background. In control trials, there was a physical difference between the two alternatives, and guppies were expected to select the larger food portion. In test trials, researchers presented two identical food portions: one encircled by a larger ring, the other by a smaller ring. In another experiment, guppies were initially trained to select the larger orange dot between two alternative ones (training phase); then, in the test phase, they were presented with two same-sized dots encircled by a large and small ring. In both cases, guppies showed a preference to select one stimulus more than chance, showing that they perceived some sort of size illusion. Of interest, the stimulus selected by guppies was the one encircled by the larger ring, the one perceived as smaller by human observers. This result suggests that 
guppies may perceive a reverse illusion. One possibility is that guppies are less sensitive to contrast (as advanced in birds by Watanabe et al., 2011). If so, they would only assimilate the object with the encircled ring. Because the object assimilated into the larger ring is likely to appear larger than the one assimilated into the smaller ring, a reverse illusion is expected to occur.

A partially similar illusion is called the Ebbinghaus illusion. Unlike the Delboeuf illusion, this is supposed to be only a "contrast" phenomenon (Massaro \& Anderson, 1971): The object surrounded by large circles contrasts with those circles (Figure 1c), appearing smaller than the other object that is less susceptible to the contrast effect with small circles. This illusion has been studied in redtail splitfin (Sovrano, Albertazzi, \& Salva, 2015). Subjects were initially trained to discriminate between two orange dots differing in size. Subsequently, they were presented the Ebbinghaus illusion. Subjects trained toward the larger dot as the positive stimulus selected the dots that appeared to be larger; similarly, subjects trained to select the smaller dot as the positive stimulus selected the one that appeared to be smaller. The authors concluded that redtail splitfin also perceive the Ebbinghaus illusory effect as a contrast phenomenon.

\section{Numerosity Illusions}

Another important class of visual illusions deals with numerosity misperception. In these cases, the quantity of stimuli presented in the visual scene is not correctly estimated because of specific spatial arrangements. For instance, items forming a single Gestalt are commonly overestimated compared with items forming smaller separate clusters, a phenomenon known as the Solitaire illusion (Frith \& Frith, 1972; see Figure 2). Recent studies showed that items located on the periphery are perceived to be $76 \%$ to $90 \%$ as numerous as centrally located items by human observers (Agrillo, Parrish, \& Beran, 2016; Pecunioso \& Agrillo, 2020). This illusion has recently been investigated in guppies. Miletto Petrazzini, Parrish, Beran, and Agrillo (2018) presented fish with two arrays made of white and black dots on a gray background. Fish were trained to select the array containing the larger amount of black dots to obtain a food reward. The following numerical contrasts were used in the training phase: 13 versus 19 dots (0.68 ratio) and 14 versus 18 dots ( 0.78 ratio). After reaching the learning criterion, guppies were shown the same numerical contrasts together with nonreinforced test trials with the illusory pattern, 16 white versus 16 black dots. In one array, black dots were centrally located and white dots were located in the perimeter; in the other array, black dots were located in the perimeter and white dots were centrally located. At the group level, guppies discriminated the 0.68 numerical ratio but did not discriminate the 0.78 ratio, in line with a robust number of studies on fish numerical abilities (reviewed in Agrillo \& Bisazza, 2018). In the presence of the Solitaire illusion, guppies demonstrated an overall misperception of numerosity in a humanlike way, as they selected more often than chance the array in which black dots were centrally located to form a single Gestalt. That said, individual analyses showed that a small percentage of subjects $(14 \%)$ actually selected this array more than chance. Hence, this type of illusion is very subtle in this species and only a few guppies are significantly susceptible to it, a fact that aligns with recent literature showing that the perception of the Solitaire illusion is almost a human characteristic, as it appears weak even to our closest relatives, such as apes (Agrillo, Parrish \& Beran, 2014a) and monkeys (Agrillo et al., 2014a; Parrish, Agrillo, Perdue, \& Beran, 2016; Parrish, Beran, \& Agrillo, 2019).

\section{Brightness Illusions}

When we misinterpret an object's brightness based on the background in which it is inserted, we incur the so-called brightness illusions. The simultaneous contrast is one of the most famous examples of this category. Even though the quantity of light reflected by each rectangle is identical, the rectangle inserted on the left in Figure 3 appears lighter than the one presented on the right, as the former is arranged in front of a lighter background and the latter is arranged in front of a darker background. According to Roe, Lu, and Hung (2005), the illusion is determined by cortical activity, particularly in the V2

Figure 2. Numerosity illusion studied in lower vertebrates: the Solitaire illusion. The same numbers of white and black dots are presented, although it seems that the centrally located items forming a single Gestalt are more numerous.

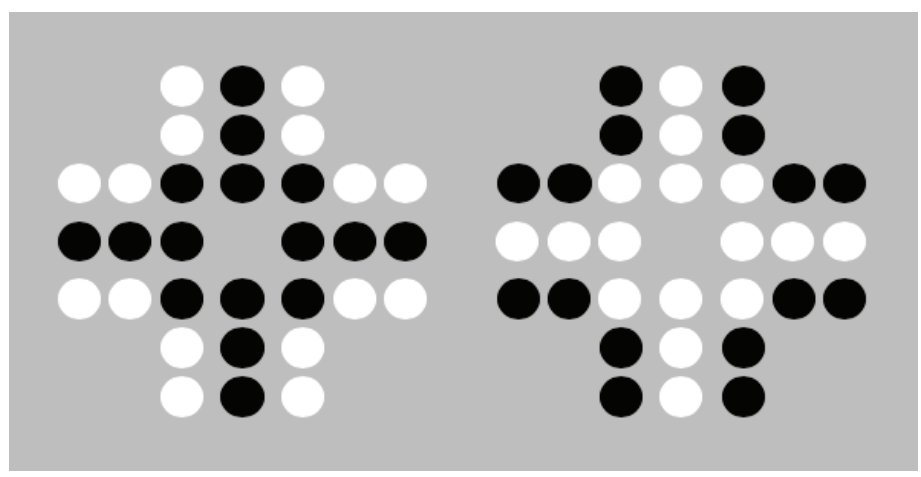



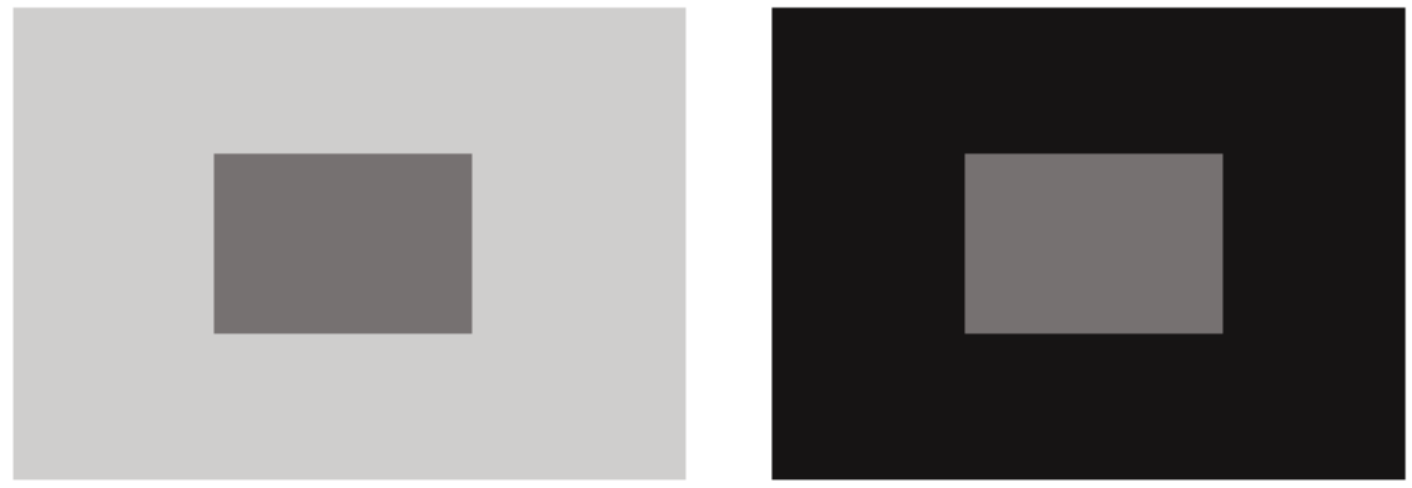

Figure 3. Brightness illusions investigated in lower vertebrates. (a) Simultaneous contrast: When two stimuli identical in lightness are presented on two different backgrounds, we tend to perceive the stimulus inserted in the lighter background as being darker.

visual area, as this would be the first place in the brain where the information about the rectangle's brightness itself would be integrated with the information coming from the surrounding background. An alternative mechanism that may play a role in this illusion's emergence is the lateral inhibition at retina level, whereby cells in one region inhibit cells in adjacent regions (Cornsweet $\&$ Teller, 1965).

This perceptual phenomenon has been investigated in guppies. Agrillo, Miletto Petrazzini, and Bisazza (2016) trained fish to discriminate between two rectangles according to their brightness. Half of the fish were trained to select the darker stimulus as positive; half were trained to select the lighter one as positive. In one condition of the training phase, the same background was used for both darker and lighter rectangles. In another condition, the lighter rectangle was presented against a lighter background, and the darker rectangle was presented against a darker background. In the last condition, the darker rectangle was presented against a lighter background, and the lighter rectangle was presented against a darker background. As soon as they reached the learning criteria, guppies started the test phase in which researchers presented the visual illusion: two identical rectangles inserted onto two different backgrounds. Most of the guppies selected the positive stimulus more than chance in the training phase, showing to have learned the task. During the test, they selected the rectangle that appeared to be darker/lighter, depending on which was the positive stimulus in the previous training phase. This has been taken as evidence of a humanlike perception of this illusory pattern (Agrillo, Miletto Petrazzini, \& Bisazza, 2016).

\section{Motion Illusions}

Susceptibility to visual illusions among freshwater fish has also been reported with respect to motion perception. Motion illusions are defined as perceptions of motion that are absent or different in the external world (Gori \& Stubbs, 2014). Among this type of illusions, the rotating snake illusion (Figure 4 ) is unquestionably one of the most popular examples. The illusion consists of a sequence of blobs with a different luminance. In particular, the specific sequence "black-dark gray-white-light gray" is misperceived in the motion-sensitive cortical area (Murakami, Kitaoka, \& Ashida, 2006), thus leading to a perception of motion that runs from the dark-tolight direction (Faubert \& Herbert, 1999). Fixational eye movements also play a key role in enhancing this illusory motion effect (Murakami et al., 2006).

Gori, Agrillo, Dadda, and Bisazza (2014) studied the susceptibility to this illusion in two teleost species, guppies and zebrafish (Danio rerio). In the training phase, the subjects were presented with a static and a dynamic object and were required to select the dynamic objects to obtain a food reward. In the test phase, the subjects were shown novel configurations to assess their ability to generalize the rule to novel stimuli, including the rotating snake illusion and a control stimulus. This control stimulus - previously used in comparative perception studies (Agrillo et al., 2015; Regaiolli et al., 2019) — was similar in terms of overall configuration, but human observers did not experience motion perception as the luminance sequences were modified. Subjects of both species were able to select the dynamic objects in the training phase; in the test phase, they both selected the rotating snake illusion more than the control stimulus, a 
fact that suggests spontaneous motion extrapolation in the presence of this physically static pattern.

\section{Illusory Contours}

Another important perceptual phenomenon that permit organisms to segregate objects from the background and easily interpret the visual scene is the formation of illusory contours. The Kanizsa figure is one of the most famous examples in this category. As shown in Figure 5a, the three Pac-Man figures are arranged so that their open angles point inward at the same area, thus generating the perception of a white triangle on a slightly darker background. Illusory perception can also be generated without the use of Pac-Man figures. An interruption of lines (Figure 5b) or phase shifting of the background can elicit the perception of an illusory figure among human observers (Figure 5c). These phenomena seem to be the result of inferences that the individual makes about objects and their spatial relationships (Gregory, 1972). Other authors have stressed the importance of depth cues in forming illusory contours that might generate the perception of a surface on the background (Coren, 1972) or the role of the brightness difference between the background and the illusory triangle (Brigner \& Gallagher, 1974).

Wyzisk and Neumeyer (2007) conducted the first study on illusory contours in fish. The authors trained goldfish (Carassius auratus) to discriminate between triangles and squares to obtain a food reward. In the test phase, subjects were shown a Kanizsa triangle and a Kanizsa square. The fish reached the visual array that resembled the stimulus associated with food reward in

Figure 4. Motion illusion investigated in lower vertebrates: Rotating snake illusion. Although the pattern is static, most humans experience rotatory motion that runs from the dark-to-light direction.

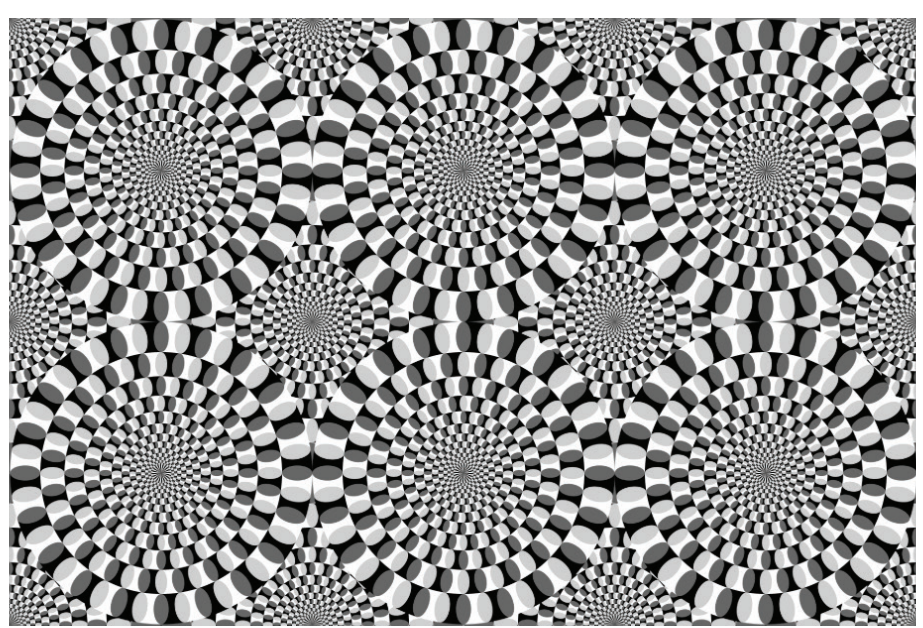

the previous training phase (e.g., the Kanizsa triangle if they were trained to select a triangle). One may argue that the choice behavior of fish in the test phase did not actually reflect the perception of illusory contours but instead might have been the result of a tendency of subjects to focus on local details (Fujita, Nakamura, Sakai, Watanabe, \& Ushitani, 2012), such as the spatial arrangements of the Pac-Man figures (e.g., an acute angle in a given position). Indeed, when illusory contours were generated by using another visual pattern (a phase shifting of the background), goldfish did not show any evidence of perceiving the illusory effect, making it difficult to draw any firm conclusion on subjective contours' perception of goldfish.

The redtail splitfin is the other teleost fish investigated in studies of illusory contours. Sovrano and Bisazza (2009) trained redtail splitfin to discriminate between a square and a triangle by using a social reward (they had to select a tunnel associated with a figure to rejoin their social companions). In the test phase, three types of illusory contours were presented: Pac-Man figures reproducing a Kanizsa triangle and Kanizsa squares, and arrays characterized by interruption or spatial phase shift of diagonal lines (again giving the subjective impression of triangles and squares to human observers). In this case, the fish were shown to perceive illusory contours with all of the stimuli. The authors hypothesized that the differential performance of goldfish with spatial phase shift of diagonal lines was not related to a true difference in perceptual mechanisms between the two species but rather to methodological differences (i.e., the use of thin diagonal lines in the goldfish study, which might have been insufficient to generate the illusory figure).

\section{Saltwater Fish}

\section{Size Illusions}

Compared to freshwater fish, very few studies have been conducted on saltwater fish. Fuss and Schluessel (2017) investigated the Delboeuf illusion and Ebbinghaus illusion in the damselfish (Chromis chromis). In the training phase, the subjects were required to discriminate between a large dot and a small dot. In the test phase, the two illusions were presented. The damselfish perceived both illusory effects in a humanlike way.

\section{Brightness Constancy}

Brightness constancy refers to the tendency to perceive an object as having the same brightness under

COMPARATIVE COGNITION \& BEHAVIOR REVIEWS 
(a)

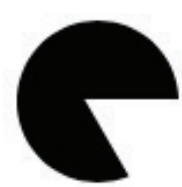

(b)

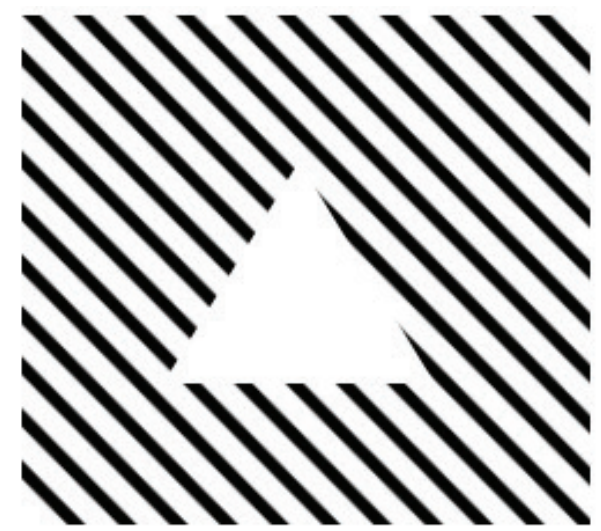

(c)

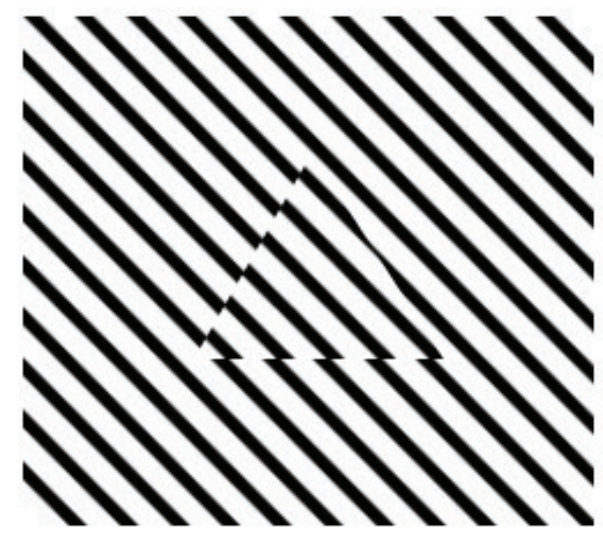

Figure 5. Illusory contours investigated in lower vertebrates. We are inclined to perceive borders even when there is no physical stimulation in order to create an easy and stable representation of the visual scene. Such illusory contours could be generated (a) with Kanizsa Pac-Man figures, (b) with an interruption of lines, or (c) by phase shifting the background.

different conditions of illumination (Hess \& Pretori, 1894). Concerning the mechanisms underlying brightness constancy, Simpson, Marshall, and Cheney (2016) investigated the coral fish (Rhinecanthus aculeatus), as known as the Picasso triggerfish. The subjects were initially required to touch with their mouth either an orange or a brown target to receive food rewards. Subsequently, these targets were embedded within more a complex scene known as the illusory cube. This stimulus presents identically colored targets that appear to human observers to be different in terms of spectral outputs on the basis of the apparent amount of illumination they are perceived to be under. In particular, the illusory cube includes shadows on some target colors. The researchers wanted to assess whether the perception of these shadows influenced how triggerfish perceived the colored targets. Subjects trained to select the orange targets as positive tended to choose the target stimulus viewed in the shadow of the illusory cube, whereas fish trained to select the brown stimulus persisted in selecting the brown targets outside the perceived shadow. Taken together, these results suggest that a coral fish takes into account the context's brightness when estimating an object's brightness, the humanlike perceptual mechanism underpinning the brightness constancy.

\section{Amphibians}

\section{Depth Illusions}

As far as we are aware, the only work on visual illusions in amphibians has been published in a conference proceedings (an abstract format). In this study, Bastakov
(1997) argued that frogs (Rana temporalis) and toads (Bufo bufo) estimate objects' distance on the basis of contextual factors. In particular, these species tend to approach small objects (as they might represent potential prey) and avoid large ones (as they might be predators). By manipulating the speed of the objects moving toward the animals, the author found that small objects moving slow were avoided by the subjects, probably because slow motion led them to overestimate the distance of the objects (and accordingly their size). Although interesting, such conclusions need further empirical evidence before being accepted as evidence of a visual illusion.

\section{Acoustic Illusions}

Sensory illusions in nonvisual modality, however, has been studied in three frog species. It is known that a sound containing brief silent gaps can be perceived as continuous if the experimenter inserts a noise into the gaps, an auditory illusion called continuity illusion (Miller \& Licklider, 1950). This phenomenon is likely to be the result of a spontaneous tendency to complete objects despite fragmentary or incomplete sensory information. It would not be adaptive if we could not grasp the meaning of a sentence in a noisy environment, as this type of fragmentary/noisy information is common in everyday life. When presented with stimuli that included complete social calls, calls with silent gaps, and calls with gaps filled with noise, Hyla chrysoscelis, $H$. chrysoscelis, and Physalaemus pustulosus did not show any clear evidence of being susceptible to this illusory phenomenon (Baugh, Ryan, Bernal, Rand, \& Bee, 2016; Seeba, Schwartz, \& Bee, 2010). 


\section{Reptiles \\ Size Illusions}

Thus far, only two studies have been published with reptiles. They both deal with size illusions, namely, the Delboeuf illusion and Müller-Lyer illusion. In the first study, Santacà, Miletto Petrazzini, Agrillo, and Wilkinson (2019) compared two species: bearded dragons (Pogona vitticeps) and red-footed tortoises (Chelonoidis carbonaria). Because, compared with mammals and birds, training reptiles seems to be difficult (Miletto Petrazzini et al., 2017), the authors used the spontaneous preference for reaching a large amount of food to investigate reptiles' susceptibility to the Delboeuf illusion. In most trials, a large piece and a small piece of food were presented on two same-sized plates. The subjects were expected to maximize food intake. Several illusory trials were also intermingled, in which the same amount of food was presented on either a large or a small plate. Bearded dragons were shown to be susceptible to the illusion, as they selected the food amount presented in the smaller plate (presumably estimated as larger). In contrast, red-footed tortoises did not select one plate more than chance, suggesting either poorer size discrimination abilities or the existence of different perceptual mechanisms between bearded dragons and red-footed tortoises. A subsequent study showed that red-footed tortoises can discriminate between the quantities of food by changing the experimental apparatus but still showed no evidence of illusory perception in the presence of the Delboeuf-like pattern (Santacà, Miletto Petrazzini, Agrillo, \& Wilkinson, 2020).

The investigation of Müller-Lyer illusions involved only bearded dragons (Santacà et al., 2020). Again, to skip the problem of how to motivate the subjects in a training task, researchers exploited their spontaneous tendency to reach the larger amount of food. In control trials, when two different-sized food sticks were presented, the subjects selected the larger one. In test trials, two same-sized food sticks were presented. Each stick was presented with two arrowheads. In one case, the arrowheads were pointed inward; in the other, they pointed outward. The food sticks and the arrowheads depicted the classical version of the Müller-Lyer illusion. In the presence of these stimuli, bearded dragons selected the food stick with arrowheads pointing inward, which appears to be longer to human observers. Taken together, the studies on the Delboeuf and Müller-Lyer illusions suggest that bearded dragons share similar perceptual mechanisms for size estimation with humans.

\section{Conclusions}

Evidence supporting a differential perception of visual illusions between mammals and birds raised the question as to whether these differences are due to the different complexity of the brain. A traditional way in the neuroscience community to compare the "complexity" of the brain among the species is in looking at the relative brain size of organisms (Marino et al., 2007; Northcutt, 2002). We accordingly focus on small-brained species to establish whether their perception of visual illusions is absent/different compared with the existing literature on "higher" vertebrates.

Even from a quick look of the literature (Figure 6), it appears clear that only a small number of lower vertebrates has been investigated. Also, the small number of studies reflects the limited number of illusory patterns tested: Zebrafish, damselfish, and triggerfish were tested with only a single pattern, whereas guppies, goldfish, and redtail splitfin were tested with two to five illusory patterns. Reptile investigation has begun only this year with only two illusory patterns; similarly, only three studies were reported in amphibians, two of them testing sensory illusions in nonvisual modality. In this sense, no conclusions could be drawn in terms of a single species on similarities with and differences from human perception. Nonetheless, we believe that some preliminary conclusions could be drawn at least by an overall view of fish literature. The susceptibility to visual illusions in fish largely resembles that of humans in the presence of different illusory phenomena, speaking against the hypothesis of a link between relative brain size and the emergence of the perceptual systems underlying visual illusions.

The fact that small-brained organisms display cognitive mechanisms described in humans is far from new in comparative psychology. Species distantly related to humans have shown impressive cognitive skills whose mechanisms were believed to be generated by larger cortical networks. Fish, for instance, have been shown to display a wide range of numerical abilities (Miletto Petrazzini, Pecunioso, Dadda, \& Agrillo, 2020), including the spontaneous ability to enumerate the number of social companions present (Dadda, Piffer, Agrillo, \& Bisazza, 2009) and the capacity to use ordinal information to find food in a series of identical locations aligned in a row (Miletto Petrazzini, Lucon-Xiccato, Agrillo, \& Bisazza, 2015). Recently, it has been advanced that fish have self-recognition, when tested in a version of the mirror test commonly used with primates (Kohda et al., 2019). Impressive cognitive abilities have also been found in invertebrates. Bees have complex numerical

COMPARATIVE COGNITION \& BEHAVIOR REVIEWS 

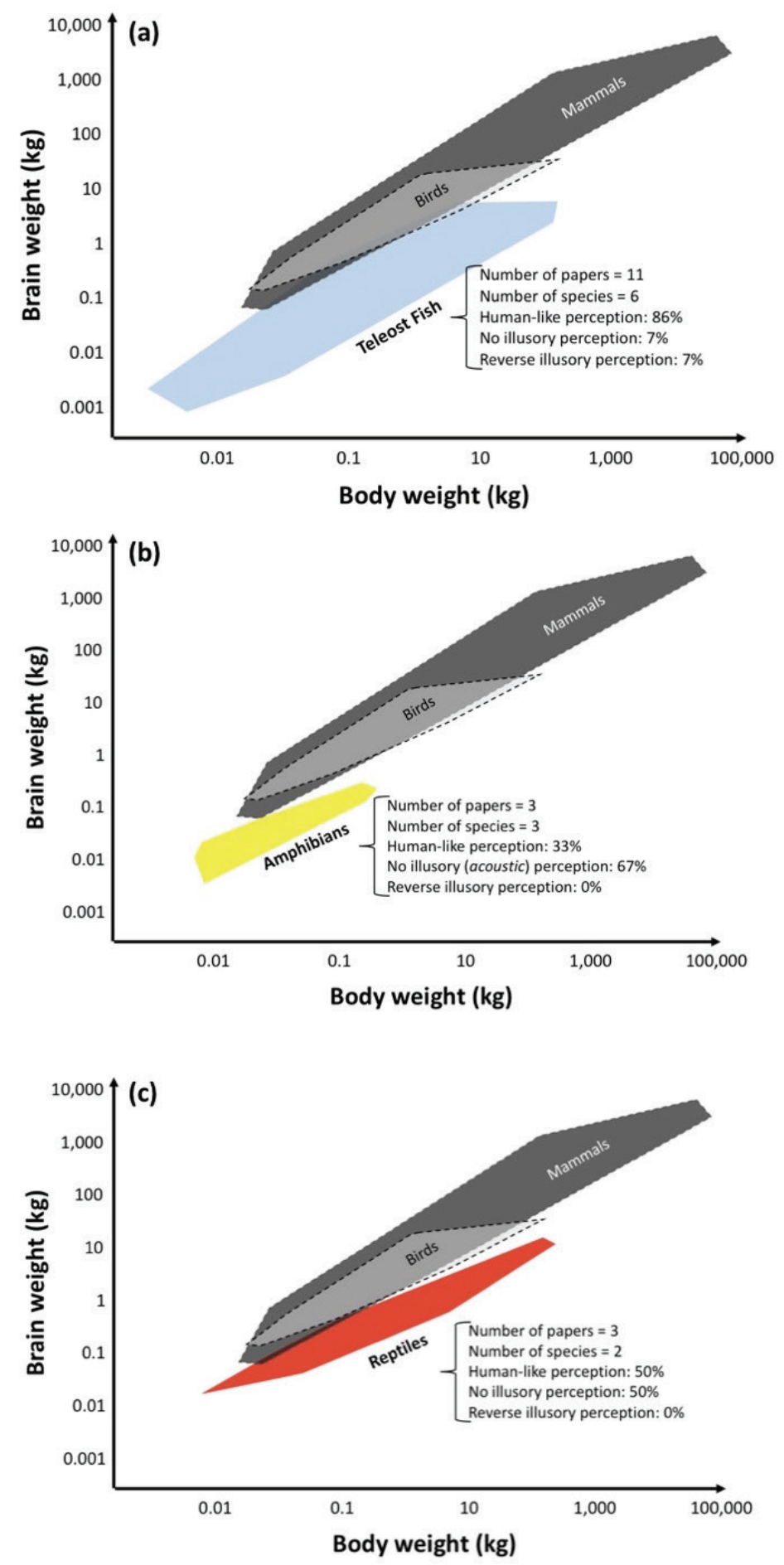

Figure 6. Brain-body relations in 623 living vertebrate species enclosed in minimum convex polygons (adapted from Jerison, 1994). As reference, data from mammals and birds are presented together with data from

(a) fish, (b) amphibians, and (c) reptiles. Fish studies suggest that similar perceptual mechanisms are shared among vertebrates regardless of their relative brain size. The pattern of data in amphibians and reptiles is more complicated and does not permit us to draw firm conclusions. This is likely also due to the limited number of studies with these two vertebrate groups.
(Bortot et al., 2019; Howard, Avarguès-Weber, Garcia, Greentree, \& Dyer, 2018) and spatial (Collett, Chittka, \& Collett, 2013) abilities and have proved to grasp abstract concepts, such as "sameness" and "difference" (Giurfa, Zhang, Jenett, Menzel, \& Srinivasan, 2001). How can these species solve such complex cognitive tasks? Part of the answer probably relies on our concept of a complex, sophisticated brain. If we continue to assume that a large network of neurons is a sine qua non condition for potentially complex tasks, we clearly incur a paradox when dealing with the outstanding performance of lower vertebrates or invertebrates. On the contrary, if we hypothesize that humans involve large neural networks in cognitive/perceptual tasks but only a few of these networks would be strictly necessary to solve the majority of the tasks, then the range of species that display a "proper" brain would be drastically larger. In line with this hypothesis, many researchers now further stress the importance of neural circuits, modularity, and interconnectivity rather than the role of relative brain size (Agrillo, 2012; Chittka \& Niven, 2009; Giurfa, 2013). A computational study by Stoianov and Zorzi (2012) provides novel insights in this field. The authors used a multilayer neural system that shares top-down and bottom-up connections - commonly called deep networks - to infer perceptions of the sensory input. The network had one visible layer encoding sensory data and two hidden layers hierarchically organized. Sensitivity to numerical information, in terms of internal coding by hidden neurons after learning, was investigated. The results showed that highest level populations of as few as 35 hidden neurons were able to support the process of numerosity estimation, definitely far fewer neurons than was previously thought (Dehaene \& Changeux, 1993). It is important to note that the response of hidden neurons was not initially stipulated; just the opposite, it represented an unsupervised emergent property. Provided that miniature brains can perform different cognitive tasks, it is only a small step to accept the idea that some perceptual laws underlying our vision can also be generated by very different nervous systems with fewer neurons. In line with this hypothesis, a recent study in honeybees (Apis mellifera) showed that even an insect brain can perceive the Ebbinghaus illusion in a humanlike way (Howard, Avarguès-Weber, Garcia, Stuart-Fox, \& Dyer, 2017).

As advanced by Chittka and Niven (2009) with respect to cognitive tasks, we can hypothesize that humans enroll large cortical networks in the perception of visual illusions, as we are equipped with greater 
replication of neuronal circuits. Such replication would add precision to sensory processes, more details to perception, and more parallel processing. However, these advantages would not produce a significant change in basic perception, thus explaining why the performance of lower vertebrates in the presence of some illusory patterns is similar.

As a last note, it is interesting to notice that nonhuman animals were also found to perceive reversed illusions: Pigeons and bantams perceived an orientation illusion in the case of Zollner illusion (Watanabe et al., 2011, 2013) but in the opposite direction compared with human and nonhuman primates (Agrillo et al., 2014b), estimating as wider the gap perceived as narrower by human observers. This illusion has been never investigated in fish, amphibians, and reptiles. This lack of studies prevents us from drawing any conclusion as to whether the perceptual mechanisms underlying objects' orientation are similar/dissimilar between mammals and other vertebrates. The only reversed illusion documented in lower vertebrates is the Delboeuf illusion (Lucon-Xiccato et al., 2019). Interestingly similar results have been found in a cartilagineous fish, the bamboo shark (Fuss \& Schluessel, 2017). There is no evidence of a reversed Delboeuf illusion in other vertebrate groups. On the other hand, pigeons experience a reversed perception of the Ebbinghaus illusion (Nakamura et al., 2008), a size illusion based on similar perceptual mechanisms. In this sense, the possibility exists that nonhuman species have similar perceptual mechanisms underlying size estimation. Such mechanisms, however, would be different from those described in humans. Even in this case, the similar perception in birds and fish of visual illusions based on a contrast effect reinforces the idea that the emergence of perceptual mechanisms underlying illusory perception is not largely dependent on the relative brain size.

\section{References}

Agrillo, C. (2012). Once upon a time there was complex numerical estimation.

Frontiers in Human. Neuroscience, 6, 300. doi:10.3389/fnhum.2012.00300

Agrillo, C., \& Bisazza, A. (2018). Understanding the origin of number sense: a review of fish studies. Philosophical Transaction of the Royal Society London B Biological Science, 373, Article 20160511. doi:10.1098/rstb.2016.0511
Agrillo, C., Gori, S., \& Beran, M. J. (2015). Do rhesus monkeys (Macaca mulatta) perceive illusory motion? Animal Cognition, 18(4), 895-910. doi:10.1007/s10071-015-0860-6

Agrillo, C., Miletto Petrazzini, M. E., \& Bisazza, A. (2016). Brightness illusions in the guppy (Poecilia reticulata). Journal of Comparative Psychology, 130(1), 55-61. doi:10.1037/com0000020

Agrillo, C., Parrish, A. E., \& Beran, M. J. (2014a). Do primates see the Solitaire illusion differently? A comparative assessment of humans (Homo sapiens), chimpanzees (Pan troglodytes), rhesus monkeys (Macaca mulatta) and capuchin monkeys (Cebus apella). Journal of Comparative Psychology, 128, 402-413. doi:10.1037/a0037499

Agrillo, C., Parrish, A. E., \& Beran, M. J. (2014b). Do rhesus monkeys (Macaca mulatta) perceive the Zollner illusion? Psychonomic Bulletin \& Review, 21, 986-994. doi:10.3758/s13423-013-0573-2

Agrillo, C., Parrish, A. E., \& Beran, M. J. (2016). How illusory is the solitaire illusion? Assessing the degree of misperception of numerosity in adult humans. Frontiers in Psychology, 7, 1663. doi:10.3389/fpsyg.2016.01663

Barbet, I., \& Fagot, J. (2002). Perception of the corridor illusion by baboons (Papio papio). Behavioural Brain Research, 132, 111-115. doi:10.1016/S0166-4328(01)00393-X

Bastakov, V. A. (1997). Visual illusions in frogs and toads. Perception, 26 (1, Suppl.), 80. doi:10.1068/v970211

Bauchot, R., Platel, R., \& Ridet, J. M. (1976). Brainbody weight relationships in Selachii. Copeia, 1976, 305-310. doi:10.2307/1443950

Baugh, A. T., Ryan, M. J., Bernal, X. E., Rand, A. S., \& Bee, M. A. (2016). Female túngara frogs do not experience the continuity illusion. Behavioral Neuroscience, 130, 62-74. doi:10.1037/bne0000115

COMPARATIVE COGNITION \& BEHAVIOR REVIEWS 
Beran, M. J. (2006). Quantity perception by adult humans (Homo sapiens), chimpanzees (Pan troglodytes) and rhesus macaques (Macaca mulatta) as a function of stimulus organization. International Journal of Comparative Psychology, 19, 386-397.

Boehm, T., Iwanami, N., \& Hess, I. (2012). Evolution of the immune system in the lower vertebrates. Annual Review of Genomics and Human Genetics, 13, 127-149. doi:10.1146/annurev-genom-090711-163747

Bortot, M., Agrillo, C., Avarguès-Weber, A., Bisazza, A., Miletto Petrazzini, M.E., \& Giurfa, M. (2019). Honeybees use absolute rather than relative numerosity in number discrimination. Biology Letters, 15(6), 20190138. doi:10.1098/rsbl.2019.0138

Brigner, W. L., \& Gallagher, M. B. (1974). Subjective contour: Apparent depth or simultaneous brightness contrast? Perception and Motor Skills, 38, 1047-1053. doi:10.2466/pms.1974.38.3c.1047

Chianese, R., Chioccarelli, T., Cacciola, G., Ciaramella, V., Fasano, S., Pierantoni, R., ... Cobellis, G. (2011). The contribution of lower vertebrate animal models in human reproduction research. General and Comparative Endocrinology, 171, 17-27. doi:10.1016/j.ygcen.2010.12.011

Chittka, L., \& Niven, J. (2009). Are bigger brains better? Current Biology, 19, R995-R1008. doi:10.1016/j.cub.2009.08.023

Collett, M., Chittka, L., \& Collett, T. S. (2013). Spatial memory in insect navigation. Current Biology, 23, R789-R800. doi:10.1016/j.cub.2013.07.020

Coren, S. (1972). Subjective contours and apparent depth. Psychology Review, 79, 359-367. doi:10.1037/h0032940

Cornsweet, T. N., \& Teller, D. (1965). Relation of increment thresholds to brightness and luminance. Journal of the Optical Society of America, 55, 13031308. doi:10.1364/JOSA.55.001303
Dadda, M., Piffer, L., Agrillo, C., \& Bisazza, A. (2009). Spontaneous number representation in mosquitofish. Cognition, 112(2), 343-348. doi:10.1016/j.cognition.2009.05.009

Dehaene, S., \& Changeux, J. P. (1993). Development of elementary numerical abilities: A neuronal model. Journal of Cognitive Neuroscience, 5, 390-407. doi:10.1162/jocn.1993.5.4.390

Demski, L. S., \& Northcutt, R. G. (1996). The brain and cranial nerves of the white shark: An evolutionary perspective. In A. P. Klimley \& D. G. Ainley (Eds.), Great white sharks. The biology of Carcharodon Carcharias (pp. 121-130). New York, NY: Academic Press. doi:10.1016/B978-012415031-7/50013-6

Eagleman, D. M. (2001). Visual illusions and neurobiology. Nature Review Neuroscience, 2(12), 920-926. doi:10.1038/35104092

Faubert, J., \& Herbert, A. M. (1999). The peripheral drift illusion: a motion illusion in the visual periphery. Perception, 28, 617-621. doi:10.1068/p2825

Frith, C. D., \& Frith, U. (1972). The solitaire illusion: An illusion of numerosity. Perception \& Psychophysics, 11, 409-410. doi:10.3758/BF03206279

Fujita, K., Blough, D. S., \& Blough, P. M. (1991). Pigeons see the Ponzo illusion. Animal Learning \& Behavior, 19, 283-293. doi:10.3758/BF03197888

Fujita, K., Nakamura, J., Sakai, A., Watanabe, S., \& Ushitani, T. (2012) Amodal completion and illusory perception in birds and primates. In O. F. Lazareva, T. Shimizu, \& E. A. Wasserman (Eds.), How animals see the world (pp. 101-116). Oxford, England: Oxford University Press. doi:10.1093/acprof:oso/9780195334654.003.0008

Fuss, T., Bleckmann, H., \& Schluessel, V. (2014). The brain creates illusions not just for us: Sharks (Chiloscyllium griseum) can "see the magic" as well. Frontiers in Neural Circuits, 8, 24. doi:10.3389/fncir.2014.00024 
Fuss, T., \& Schluessel, V. (2017). The Ebbinghaus illusion in the gray bamboo shark (Chiloscyllium griseum) in comparison to the teleost damselfish (Chromis chromis). Zoology, 123, 16-29. doi:10.1016/j.zool.2017.05.006

Giurfa, M. (2013). Cognition with few neurons: Higherorder learning in insects. Trends in Neurosciences, 36(5), 285-294. doi:10.1016/j.tins.2012.12.011

Giurfa, M., Zhang, S., Jenett, A., Menzel, R., \& Srinivasan, M. V. (2001). The concepts of "sameness" and "difference" in an insect. Nature, 410(6831), 930-933. doi:10.1038/35073582

Gori, S., Agrillo, C., Dadda, M., \& Bisazza, A. (2014). Do fish perceive illusory motion? Scientific Reports, 4, 6443. doi:10.1038/srep06443

Gori, S., Molteni, M., \& Facoetti, A. (2016). Visual illusions: An interesting tool to investigate developmental dyslexia and autism spectrum disorder. Frontiers in Human Neuroscience, 10, 175. doi:10.3389/fnhum.2016.00175

Gori, S., \& Stubbs, D. A. (2014). Motion illusions as a psychophysical tool to investigate the visual system. In A. Geremek, M. W. Greenlee, \& S. Magnussen (Eds.), Perception beyond Gestalt: Progress in vision research (pp. 128-143). New York, NY: Psychology Press.

Gregory, R. L. (1963). Distortion of visual space as inappropriate constancy scaling. Nature, 119, 678-680. doi:10.1038/199678a0

Gregory, R. L. (1972). Cognitive contours. Nature, 238, 51-52. doi:10.1038/238051a0

Gregory, R. L. (1997). Visual illusions classified. Trends in Cognitive Sciences, 1, 190-194. doi:10.1016/S1364-6613(97)01060-7

Hess, C., \& Pretori, H. (1894). Quantitative investigation of the lawfulness of simultaneous brightness contrast. Albrecht von Graefe Archiv fur Ophthalmologie, 40, 1-24. doi:10.1007/BF01693963
Howard, S. R., Avarguès-Weber A, Garcia, J. E., Greentree, A. D., \& Dyer, A. G. (2018). Numerical ordering of zero in honeybees. Science, 360, 11241126. doi:10.1126/science.aar4975

Howard, S. R., Avarguès-Weber, A., Garcia, J., StuartFox, D., \& Dyer, A. G. (2017). Perception of contextual size illusions by honeybees in restricted and unrestricted viewing conditions. Proceedings of the Royal Society of London B, 284, 20172278. doi:10.1098/rspb.2017.2278

Howe, C. Q., \& Purves, D. (2005). The Müller-Lyer illusion explained by the statistics of image-source relationships. Proceedings of the National Academy of Sciences of the United States of America, 102(4), 1234-1239. doi:10.1073/pnas.0409314102

Jerison, H. J. (1991). Brain size and the evolution of mind. New York, NY: American Museum of Natural History.

Jerison, H. J. (1994). Evolution of the brain. In D. W. Zaidel (Ed.), Neuropsychology (pp. 53-81). San Diego, CA: Academic Press. doi:10.1016/B978-0-08-092668-1.50009-0

King, D. L. (1988). Assimilation is due to one perceived whole and contrast is due to two perceived wholes. New Ideas in Psychology, 6(3), 277-288. doi:10.1016/0732-118X(88)90039-6

Kohda, M., Hotta, T., Takeyama, T., Awata, S., Tanaka, H., Asai, J-y, \& Jordan, A. L. (2019). If a fish can pass the mark test, what are the implications for consciousness and self-awareness testing in animals? PLOS Biology, 17(2), e3000021. doi:10.1371/journal.pbio.3000021

Lotto, R. B., \& Purves, D. (2002). The empirical basis of color perception. Consciousness and Cognition, 11, 609-629. doi:10.1016/S1053-8100(02)00014-4

Lucon-Xiccato, T., Miletto Petrazzini, M. E., Agrillo, C., \& Bisazza, A. (2015). Guppies (Poecilia reticulata) discriminate among two quantities of food items but prioritise item size over total amount. Animal Behaviour, 107, 183-191. doi:10.1016/j.anbehav.2015.06.019 
Lucon-Xiccato, T., Santacà, M., Miletto Petrazzini, M.E., Agrillo, C., \& Dadda, M. (2019). Guppies, Poecilia reticulata, perceive a reversed Delboeuf illusion. Animal Cognition, 22(3), 291-303. doi:10.1007/s10071-019-01237-6

Marino, L., Connor, R. C., Fordyce, R. E., Herman, L. M., Hof, P. R., Lefevbre, L., ... Whitehead, H. (2007). Cetaceans have complex brains for complex cognition. PLoS Biology, 5(6), e139. doi:10.1371/journal.pbio.0050139

Massaro, D. W., \& Anderson, N. H. (1971). Judgemental model of the Ebbinghaus illusion. Journal of Experimental Psychology, 89, 147-151. doi:10.1037/h0031158

Miletto Petrazzini, M. E., Parrish, A. E., Beran, M. J., \& Agrillo, C. (2018). Exploring the solitaire illusion in guppies (Poecilia reticulata). Journal of Comparative Psychology, 132(1), 48-57. doi:10.1037/com0000092

Miletto Petrazzini, M. E., Pecunioso, A., Dadda, M., $\&$ Agrillo, C. (2020). Searching for the critical $p$ of Macphail's null hypothesis: The contribution of numerical abilities of fish. Frontiers in Psychology, 11, 55. doi:10.3389/fpsyg.2020.00055

Miletto Petrazzini, M. E., Fraccaroli, I., Gariboldi, F., Agrillo, C., Bisazza, A., Bertolucci, C., \& Foà, A. (2017). Quantitative abilities in a reptile (Podarcis sicula). Biology Letters, 13, 20160899. doi:10.1098/rsbl.2016.0899

Miletto Petrazzini, M. E., Lucon-Xiccato, T., Agrillo, C., \& Bisazza, A. (2015). Use of ordinal information by fish. Scientific Reports, 5 . doi:10.1038/srep15497

Miller, G. A., \& Licklider, J. C. R. (1950). The intelligibility of interrupted speech. Journal of the Acoustical Society of America, 22, 167-173. doi:10.1121/1.1906584

Murakami, I., Kitaoka, A., \& Ashida, H. (2006). A positive correlation between fixation instability and the strength of illusory motion in a static display. Vision Research, 46, 2421-2431. doi:10.1016/j.visres.2006.01.030
Nakamura, N., Watanabe, S., \& Fujita, K. (2008). Pigeons perceive the Ebbinghaus-Titchener circles as an assimilation illusion. Journal of Experimental Psychology: Animal Behavior Processes, 34(3), 375-387. doi:10.1037/0097-7403.34.3.375

Northcutt, R. G. (1997). Evolution of gnathostome lateral line ontogenies. Brain, Behavior and Evolution, 50, 25-37. doi:10.1159/000113319

Northcutt, R. G. (2002). Understanding vertebrate brain evolution. Integrative Comparative Biology, 42, 743-756. doi:10.1093/icb/42.4.743

Notredame, C. E., Pins, D., Deneve, S., \& Jardri, R. (2014). What visual illusions teach us about schizophrenia. Frontiers in Integrative Neuroscience, 8,63. doi:10.3389/fnint.2014.00063

Parrish, A. E., Agrillo, C., Perdue, B. M., \& Beran, M. J. (2016). The elusive illusion: Do children (Homo sapiens) and capuchin monkeys (Cebus apella) see the Solitaire illusion? Journal of Experimental Child Psychology, 142, 83-95. doi:10.1016/j.jecp.2015.09.021

Parrish, A. E., \& Beran, M. J. (2014). When less is more: like humans, chimpanzees (Pan troglodytes) misperceive food amounts based on plate size. Animal Cognition, 17(2), 427-434. doi:10.1007/s10071-013-0674-3

Parrish, A. E., Beran, M. J., \& Agrillo, C. (2019). Linear numerosity illusions in capuchin monkeys (Sapajus apella), rhesus macaques (Macaca mulatta), and humans (Homo sapiens). Animal Cognition, 22(5), 883-889. doi:10.1007/s10071-019-01288-9

Parrish, A. E., Brosnan, S. F., \& Beran, M. J. (2015). Do you see what I see? A comparative investigation of the Delboeuf illusion in humans (Homo sapiens), rhesus monkeys (Macaca mulatta), and capuchin monkeys (Cebus apella). Journal of Experimental Psychology: Animal Learning and Cognition, 41, 395-405. doi:10.1037/xan0000078

Pecunioso, A., \& Agrillo, C. (2020). Do professional musicians perceive numerosity illusions differently? Psychology of Music. Advance online publication. https://doi:10.1177/0305735619888804 
Pepperberg, I. M., Vicinay. J., \& Cavanagh, P. (2008). The Muller-Lyer illusion is processed by a Grey parrot (Psittacus erithacus). Perception, 37, 765-781. doi:10.1068/p5898

Pessoa, V. F., Monge-Fuentes, V., Simon, C. Y., Suganuma, E., \& Tavares, M. C. H. (2008). The Müller-Lyer illusion as a tool for schizophrenia screening. Reviews in the Neurosciences, 19, 91-100. doi:10.1515/REVNEURO.2008.19.2-3.91

Regaiolli, B., Rizzo, A., Ottolini, G., Miletto Petrazzini, M. E., Spiezio, C., \& Agrillo, C. (2019). Motion illusions as environmental enrichment for zoo animals: A preliminary investigation on lions (Panthera leo). Frontiers in Psychology, 10, 2220. doi:10.3389/fpsyg.2019.02220

Roe, A. W., Lu, H. D., \& Hung, C. P. (2005). Cortical processing of a brightness illusion. Proceedings of the National Academy of Sciences of the United States of America, 102, 3869-3874. doi:10.1073/pnas.0500097102

Rosa Salva, O., Rugani, R., Cavazzana, A., Regolin, L., \& Vallortigara, G. (2013). Perception of the Ebbinghaus illusion in four-day-old domestic chicks (Gallus gallus). Animal Cognition, 16, 895-906. doi:10.1007/s10071-013-0622-2

Santacà, M., \& Agrillo, C. (2020). Perception of the Müller-Lyer illusion in guppies (Poecilia reticulata). Current Zoology. Advance online publication. doi:10.1093/cz/zoz041

Santacà, M., Miletto Petrazzini, M. E., Agrillo, C., \& Wilkinson, A. (2019). Can reptiles perceive visual illusions? Delboeuf illusion in red-footed tortoise (Chelonoidis carbonaria) and bearded dragon (Pogona vitticeps). Journal of Comparative Psychology, 133(4), 419-427. doi:10.1037/com0000176

Santacà, M., Miletto Petrazzini, M. E., Agrillo, C., \& Wilkinson, A. (2020). Exploring the Müller-Lyer illusion in a nonavian reptile (Pogona vitticeps). Journal of Comparative Psychology. Advance online publication. doi:10.1037/com0000222
Santacà, M., Miletto Petrazzini, M. E., Agrillo, C., \& Wilkinson, A. (under review). Visual illusions in a non-avian reptile eye: Lizards (Pogona vitticeps) perceive the Müller-Lyer illusion. Journal of Comparative Psychology.

Seeba, F., Schwartz, J. J., \& Bee, M. A. (2010). Testing an auditory illusion in frogs: Perceptual restoration or sensory bias? Animal Behaviour, 79, 1317-1328. doi:10.1016/j.anbehav.2010.03.004

Simpson, E. E., Marshall, N. J., \& Cheney, K. L. (2016). Coral reef fish perceive lightness illusions. Scientific Reports, 6, 35335. doi:10.1038/srep35335

Sneddon, L. U. (2004) Evolution of nociception in vertebrates: Comparative analysis of lower vertebrates. Brain Research Reviews, 46, 123-130. doi:10.1016/j.brainresrev.2004.07.007

Sovrano, V. A., Albertazzi, L., \& Salva, O. R. (2015). The Ebbinghaus illusion in a fish (Xenotoca eiseni). Animal Cognition, 18(2), 533-542. doi:10.1007/s10071-014-0821-5

Sovrano, V. A., \& Bisazza, A. (2009). Perception of subjective contours in fish. Perception, 38(4), 579-590. doi:10.1068/p6121

Sovrano, V. A., Da Pos, O., \& Albertazzi, L. (2016). The Müller-Lyer illusion in the teleost fish Xenotoca eiseni. Animal Cognition, 19(1), 123-132. doi:10.1007/s10071-015-0917-6

Stoianov, I., \& Zorzi, M. (2012). Emergence of a 'visual number sense' in hierarchical generative models. Nature Neuroscience, 15, 194-196. doi:10.1038/nn.2996

Suganuma, E., Pessoa, V. F., Monge-Fuentes, V., Castro, B. M., \& Tavares, M. C. H. (2007). Perception of the Müller-Lyer illusion in capuchin monkeys (Cebus apella). Behavioural Brain Research, 182, 67-72. doi:10.1016/j.bbr.2007.05.014

van Dongen, P. A. M. (1998). Brain size in vertebrates. In R. Nieuwenhuys, H. J. ten Donkelaar, \& C. Nicholson (Eds.), The central nervous system of vertebrates (Vol. 3, pp. 2099-2134). Berlin: Springer. doi:10.1007/978-3-642-18262-4_23 
Ward, L. M., Porac, C., Coren, S., \& Girgus, J.S. (1977). The case for misapplied constancy scaling: Depth associations elicited by illusion configurations. The American Journal of Psychology, 90(4), 609-620. doi:10.2307/1421735

Watanabe, S., Nakamura, N., \& Fujita, K. (2011). Pigeons perceive a reversed Zöllner illusion. Cognition, 119, 137-141. doi:10.1016/j.cognition.2010.10.020
Watanabe, S., Nakamura, N., \& Fujita, K. (2013). Bantams (Gallus gallus domesticus) also perceive a reversed Zöllner illusion. Animal Cognition, 16, 109-115. doi:10.1007/s10071-012-0556-0

Wyzisk, K., \& Neumeyer, C. (2007). Perception of illusory surfaces and contours in goldfish. Visual Neuroscience, 24, 291-298. doi:10.1017/S095252380707023X 